\title{
ANÁLISE TERMOECONÔMICA DE COGERAÇÃO COM RESÍDUOS DA PALMA
}

\section{THERMOECONOMIC ANALYSIS OF COGENERATION WITH PALM BIOMASS}

\author{
H. F. A. F. REIS ${ }^{1}$, R. PEREZ ${ }^{2}$ \\ ${ }^{1}$ Universidade Federal de Viçosa, Departamento de Engenharia Química \\ ${ }^{2}$ Universidade Federal de Viçosa, Departamento de Tecnologia de Alimentos \\ E-mail: heytor.frauches@ufv.br
}

articleinfo

Article history:

Received 2017-04-25

Accepted 2017-07-17

Available online 2017-11-20
PALAVRAS-CHAVE: Exergia; Exergoeconômia; Termoeconomia; Palma; Cogeração.

KEYWORDS: Exergy; Exergoeconomic; Thermoeconomic; Palm; Cogeneration.

RESUMO: A cultura da palma representa mais de um terço da produção mundial de óleos vegetais. $O$ processamento dos frutos da palma demanda grande quantidade de eletricidade e vapor, entretanto, gera biomassa residual com elevado conteúdo energético. Devido a essas características, a cogeração é empregada para reduzir custos operacionais. $O$ objetivo desse estudo foi avaliar os custos de produção de vapor e eletricidade via cogeração de resíduos da palma utilizando a metodologia SPECO. A unidade de cogeração foi dimensionada para atender a demanda energética de uma planta de extração de óleo com capacidade de processamento de 50 toneladas de cachos de palma por hora. Os custos obtidos para o vapor e eletricidade foram, respectivamente, de 12,18 R\$/ton e 124,12 R\$/MWh. Ao se comparar o custo de produção da energia com o custo de sua compra, constatou-se que a implantação da cogeração pode gerar uma economia anual de $R \$ 4.795 .083,92$ para a unidade de processamento de palma deste estudo.

\begin{abstract}
The palm culture represents more than one third of global vegetable oil production. The palm processing demands large amounts of electricity and steam, however, produces biomass with high energetic potential. Because of these characteristics, cogeneration is applied in order to decrease operational costs. The goal of this study was to evaluate the production costs of steam and electricity produced by cogeneration of palm residues, utilizing SPECO methodology. The cogeneration plant was dimensioned to supply the energetic demand of a mill which process 50 tons of palm bunches per hour. The costs obtained for steam and electricity was, respectively, $12.18 \mathrm{R} \$ /$ ton e $124.12 \mathrm{R} \$ / \mathrm{MWh}$. When a comparison is done between production cost and purchase price of electricity, results shows that cogeneration could generate an economy of $R \$ 4,795,083.92$ per year for the palm mill of this study.
\end{abstract}

\section{INTRODUÇÃO}

A cogeração é largamente utilizada em plantas de beneficiamento de palma devido à disponibilidade de biomassa residual e à demanda de vapor e eletricidade para processos. $\mathrm{O}$ que possibilita a redução de custos operacionais e em alguns casos a geração de receita pela comercialização de energia excedente. 
A palma de óleo é a principal cultura oleaginosa do mundo, seus frutos produzem dois tipos diferentes de óleos: palma, extraído do mesocarpo, e palmiste, extraído da semente. A estimativa para a produção na safra 2016/2017 é de 64,5 milhões de toneladas de óleo de palma e 7,64 milhões de toneladas de palmiste, que representaria $38,82 \%$ da produção global de óleos vegetais (USDA, 2016). Ainda segundo as estimativas da USDA (2016), os principais produtores são a Indonésia e a Malásia que representam, respectivamente, 53,44\% e $32,06 \%$ da produção mundial, enquanto o Brasil é apenas o $12^{\circ}$ produtor com $0,52 \%$ de participação.

De acordo com Cespedes e Junior (1997) a análise exergética consiste na utilização concomitante da $1^{\mathrm{a}}$ e $2^{\mathrm{a}}$ Lei da Termodinâmica na avaliação da eficiência de processos. Enquanto, de forma complementar, a análise exergoeconômica permite estimar os custos unitários de produtos como vapor e eletricidade, e de perdas monetárias devido às irreversibilidades (KWAK et al., 2003).

Esse estudo aplica os conceitos de termoeconomia, mais especificamente, a análise exergoeconômica à um projeto de cogeração com biomassa residual da palma. Com o objetivo de mensurar os custos de geração de vapor e eletricidade e o impacto da cogeração na viabilidade em uma planta de beneficiamento de palma.

\section{REVISÃO DE LITERATURA}

\subsection{Demanda Energética e Produção de Biomassa Residual}

O processamento da palma gera biomassa residual com elevado potencial energético. A Tabela 1 apresenta uma comparação da produção de biomassa e dos consumos específicos de vapor e eletricidade para o processamento, segundo alguns autores, em termos relativos à massa de cachos de frutos frescos (CFF) processada.

Tabela 1: Produção de biomassa residual e demanda energética por unidade de CFF.

\begin{tabular}{ccccc}
\hline \multicolumn{5}{c}{ Produção de resíduos } \\
\hline $\begin{array}{c}\text { Cachos } \\
(\% \text { CFF })\end{array}$ & $\begin{array}{c}\text { Fibras } \\
(\% \mathrm{CFF})\end{array}$ & $\begin{array}{c}\text { Cascas } \\
(\% \mathrm{CFF})\end{array}$ & $\begin{array}{c}\text { Efluentes Líquidos } \\
(\% \mathrm{CFF})\end{array}$ & Referência \\
\hline 22 & 12 & 5 & 50 & $($ JÚNIOR, 2006) \\
$20,26-$ & $13,28-$ & $3,23-$ & - & (ARRIETA et al., 2007) \\
24,00 & 13,75 & 5,00 & 67 & (YUSOFF, 2006) \\
22 & 13,5 & 5,5 & 87 & (PRASERTSAN e \\
$20-30$ & $12-13$ & $6,8-7,4$ & PRASERTSAN, 1996) \\
\hline \multicolumn{5}{c}{ Demanda energética } \\
\hline Vapor (kg/ton CFF) & Eletricidade (kWh/ton CFF) & Referência \\
\hline \multicolumn{5}{c}{528} \\
$550-750$ & \multicolumn{3}{c}{ 17-38 } & (HUSAIN et al., 2002) \\
\hline
\end{tabular}




\subsection{Termoeconomia e Análise Exergoeconômica}

Segundo Moran e Shapiro (2009) o termo termoeconomia é utilizado de maneira geral em análises de sistemas térmicos, onde são aplicados conhecimentos de engenharia e termodinâmica, associados à engenharia econômica. A análise em termos de exergia ao invés de energia é descrita na literatura (KEÇEBAŞ, 2013; KWAK et al., 2003; ROSEN e DINCER, 2003) como a forma mais correta de mensurar o desempenho desses sistemas e atribuir valor econômico aos seus produtos. Sendo a exergia definida como o limite teórico de energia disponível para realizar trabalho que é obtido de um sistema, quando este atinge o equilíbrio termodinâmico com o ambiente (TSATSARONIS, 2007).

Existem algumas metodologias propostas para realizar análises termoeconômicas a partir da exergia. Entretanto, segundo Abusoglu e Kanoglu (2009) podem ser divididas em duas categorias: métodos de contabilidade exergoeconômica (algébricos) e métodos Lagrangianos (cálculo).

Os métodos algébricos utilizam equações lineares para mensurar os custos dos produtos, através da avaliação dos componentes e do sistema. As duas principais abordagens desse método são a modified productive structure analysis (MOPSA) e specific exergy costing (SPECO). MOPSA foi utilizada primeiramente por KIM et al. (1998) e é baseada na teoria dos custos exergéticos de Lozano e Valero (1993). Enquanto SPECO, abordagem utilizada neste trabalho, foi desenvolvida e aperfeiçoada por Lazzaretto e Tsatsaronis $(1999,2006)$. A principal diferença é que a abordagem SPECO necessita de equações auxiliares que correspondem a critérios de partição de custos, o que confere maior liberdade à análise.

Já as abordagens Lagrangianas apresentam maior complexidade, pois são baseadas em equações diferencias e tem como objetivo calcular os custos marginais e realizar a otimização de todo o sistema (ABUSOGLU; KANOGLU, 2009; LAZZARETTO; TSATSARONIS, 2006).

\section{METODOLOGIA}

Antes de aplicar a análise termoeconômica, foram realizados os balanços de massa e energia da planta de cogeração. Em regime permanente o balanço de massa no volume de controle (VC) é descrito pela Equação (1).

$$
\sum M e-\sum M s=0
$$

Em que $M e$ e $M s$ são, respectivamente, as vazões mássicas entrando e saindo do VC.

Para um volume de controle em regime permanente, desprezando as variações de energia cinética e potencial, a primeira lei da termodinâmica pode ser escrita como a Equação (2).

$$
Q v c-W v c+\sum M e H e-\sum M s H s=0
$$


Onde $Q v c$ é a taxa de transferência de calor e $W v c$ a potência transferida através do VC. Enquanto $H e$ e $H s$ são as entalpias específicas das correntes de entrada e de saída.

Para realizar a análise exergética, a Equação de balanço de exergia para sistemas não adiabáticos (3) (KWAK et al., 2003) foi aplicada de forma simplificada.

$$
\Delta E q+\Delta E f+\Delta E d=E w
$$

Onde $\Delta E q$ é a taxa de variação de exergia química, a qual usualmente é relevante para os combustíveis. A exergia química $E q$ do combustível foi obtida, através da Equação (4), pelo produto da vazão mássica $M b$ e da exergia especifica da biomassa da palma $E b$, a qual é encontrada pelas Equações (5) e (6) (SZARGUT et al., 1988).

$$
\begin{aligned}
& E q=M b \times E b \\
& \beta=\frac{1,0412+0,2160 \frac{Z H 2}{Z C}\left(1+0,7884 \frac{Z H 2}{Z C}\right)-0,0450 \frac{Z N 2}{Z C}}{1-0,3035 \frac{Z O 2}{Z C}} \\
& E b=\beta(P C I b+L w Z w)+b w Z w
\end{aligned}
$$

Os termos $Z H 2, Z C, Z N 2$ e ZO2 se referem à fração mássica elementar; $P C I b$ ao poder calorífico inferior e $Z_{W}$ ao teor de umidade da biomassa. Enquanto os termos $L w$ e $b w$ são, respectivamente, a entalpia de vaporização $(2442 \mathrm{~kJ} / \mathrm{kg})$ e exergia química da água $(50 \mathrm{~kJ} / \mathrm{kg})$.

$\mathrm{O}$ segundo termo da Equação (3) corresponde à variação de exergia física no VC devido aos fluxos de massa entrando $\Delta E f e$ e saindo $\Delta E f s$, conforme a Equação (7). A exergia física é composta da exergia térmica e mecânica que estão associadas, respectivamente, ao gradiente de temperatura e de pressão entre o sistema e o ambiente.

$$
\Delta E f=\left(\sum E f e-\sum E f s\right)
$$

Já o termo $E d$ é obtido da Equação (8) e mensura a taxa de destruição de exergia devido à transferência de calor $Q v c$ e variação de entropia dos fluxos entrando $S e$ e saindo $S s$ do $\mathrm{VC}$, em que $T o$ é a temperatura do ambiente, adotada como $25^{\circ} \mathrm{C}$. Por fim, $E w$ mensura a exergia transmitida na forma de trabalho no eixo ou eletricidade.

$$
E d=T o\left(\sum S e-\sum S s+\frac{Q v c}{T o}\right)
$$

$\mathrm{Na}$ análise exergoeconômica utilizou-se a Equação (9), referente à metodologia SPECO (LAZZARETTO; TSATSARONIS, 2006).

$$
\left(\sum E e \times c e-\sum E s \times c s\right)+E q \times c q+Z=E w \times c w
$$


Os termos ce e $c s$ são os custos exergéticos específicos dos fluxos de massa, $c q$ o custo exergético do calor e $c w$ o custo do trabalho ou eletricidade transferido pelo VC.

O método do custo anualizado (MORAN, 1982) foi utilizado para estimar os custos operacionais e capitais dos equipamentos $Z(R \$ / S)$ através das Equações $(10,11,12$ e 13).

$$
\begin{aligned}
& V p=I-\frac{V r}{(1+r)^{N}} \\
& F R C=\left(\frac{r(1+r)^{N}}{(1+r)^{N}-1}\right) \\
& C=V p \times F R C \\
& Z=\frac{\varphi C}{3600 t}
\end{aligned}
$$

Onde $V p(\mathrm{R} \$)$ é o valor presente, $I(\mathrm{R} \$)$ o investimento, $V r(\mathrm{R} \$)$ o valor residual, $r$ a taxa de amortização, $n$ (anos) o período de amortização, $F R C$ o fator de recuperação de capital, $C$ (R\$/ano) o custo anual equivalente, $\varphi$ o fator de custo de manutenção e $t$ (horas/ano) o tempo de operação.

Os cálculos foram desenvolvidos com auxílio do software Engineering Equation Solver (EES), desenvolvido por Klein e Alvarado (1995).

\section{RESULTADOS E DISCUSSÃO}

\subsection{Considerações para a Análise}

O dimensionamento da planta de cogeração partiu da demanda de vapor para o processamento da palma. Considerou-se que o consumo específico de vapor da planta é de $600 \mathrm{~kg} /$ ton CFF e que o vapor demandado seria saturado a 3 bar (HUSAIN et al., 2002).

Serão utilizadas na cogeração apenas as fibras e cascas, na proporção mássica de 2:1. Os engaços não são utilizados, pois apresentam vantagens como cobertura de solo e, além disso, as fibras e cascas já fornecem a energia necessária para o processamento (JÚNIOR, 2006).

Para o cálculo da exergia do combustível, via as Equações (5) e (6), foram utilizados os dados de composição elementar apresentada na Tabela 2. Para as fibras o teor de umidade utilizado foi de $37,2 \%$ e PCI de $11,48 \mathrm{MJ} / \mathrm{kg}$, enquanto os respectivos valores para as cascas são $21,4 \%$ de umidade e $14.55 \mathrm{MJ} / \mathrm{kg}$ (Uemura et al., 2011). Os valores de exergia específica encontrados foram de $14.007 \mathrm{~kJ} / \mathrm{kg}$ para as fibras e $17.018 \mathrm{~kJ} / \mathrm{kg}$ para as cascas.

O sistema de cogeração, ilustrado de forma simplificada na Figura (1), é composto por uma caldeira aquatubular, tubulações de aço carbono com isolamento térmico para transporte do vapor e uma turbina de ação. 
Tabela 2: Composição Elementar das biomassas residuais da palma.

\begin{tabular}{lllll}
\hline \multirow{2}{*}{ Biomassa } & \multicolumn{5}{c}{ Fração mássica em base seca (\%) } \\
& $\mathrm{C}$ & $\mathrm{O}$ & $\mathrm{H}$ & $\mathrm{N}$ \\
\hline Fibras & 46,92 & 42,66 & 5,89 & 1,12 \\
Cascas & 46,68 & 36,70 & 6,00 & 1,40 \\
\hline
\end{tabular}

Fonte: Uemura et al. (2011).

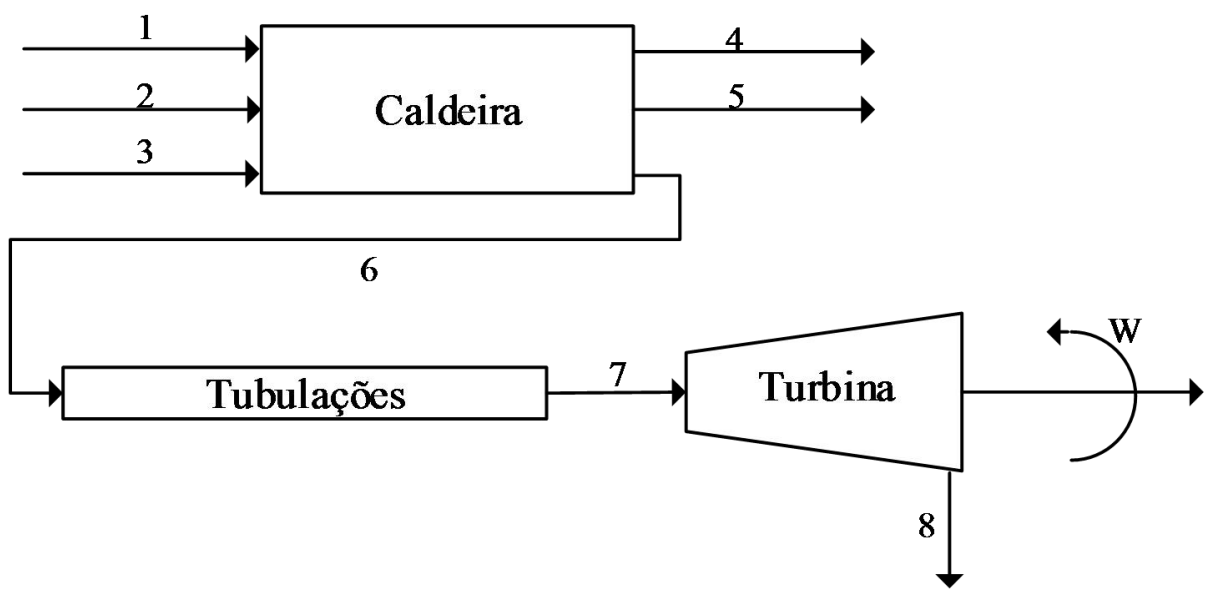

Figura 1: Fluxograma simplificado do sistema de cogeração.

A Tabela 3 especifica os fluxos mássicos do sistema e as respectivas exergias totais $(E)$, as quais representam o somatório da exergia química e física para cada corrente. A corrente 1 é referente ao fluxo de ar entrado no pré-aquecedor e a 5 aos gases de exaustão da chaminé. O fluxo 3 é a água que escoa para o desaerador da caldeira e 8 é o vapor que será utilizado no processamento da palma.

Tabela 3: Especificações das correntes mássicas do sistema.

\begin{tabular}{cccccc}
\hline Corrente & Especificação & $\mathrm{M}(\mathrm{kg} / \mathrm{s})$ & $\mathrm{T}\left({ }^{\circ} \mathrm{C}\right)$ & $\mathrm{P}($ bar $)$ & $E(\mathrm{~kW})$ \\
\hline 1 & Ar $^{1}$ & 14,953 & 25 & 1 & 0,00 \\
2 & Combustível & 2,798 & 25 & 1 & $42.001,27$ \\
3 & Água & 8,333 & 25 & 1 & 0,00 \\
4 & Cinzas & 0,278 & 25 & 1 & 0,00 \\
5 & Gases & 17,473 & 120 & 1 & $1.228,09$ \\
6 & Vapor & 8,333 & 420 & 42 & $10.258,05$ \\
7 & Vapor & 8,333 & 418,6 & 41,47 & $10.232,19$ \\
8 & Vapor & 8,333 & 142,9 & 3 & $5.422,80$ \\
\hline
\end{tabular}

\footnotetext{
${ }^{1}$ Por recomendação do fabricante é adotado $30 \%$ de excesso de ar para a combustão.
} 
A Tabela 4 apresenta os balanços exergéticos para cada componente e para todo o sistema, obtidos pela aplicação da Equação (3). A parcela de exergia química é a exergia total entregue pelo combustível à caldeira e a última coluna representa a exergia destruída devida as irreversibilidades dos processos, conforme a Equação (7). O termo de trabalho consumido na caldeira é referente ao funcionamento das motobombas de água, sopradores de fuligem e ventiladores.

Tabela 4: Balanço exergético.

\begin{tabular}{llllc}
\hline Componente & $E w(\mathrm{~kW})$ & $\Delta E q(\mathrm{~kW})$ & $\Delta E f(\mathrm{~kW})$ & $\Delta E d(\mathrm{~kW})$ \\
\hline Caldeira & $-220,65$ & $42.001,27$ & $-11.486,14$ & $-30.735,78$ \\
Tubulações & 0,00 & 0,00 & 25,86 & $-25,86$ \\
Turbina & $3.608,34$ & 0,00 & $4.809,38$ & $-1.201,04$ \\
Global & $3.387,69$ & $42.001,27$ & $-6.650,90$ & $-31.962,68$ \\
\hline
\end{tabular}

Percebe-se pela análise das Tabelas 3 e 4 que há uma geração líquida de 3.387,69 kW de energia elétrica e exportação de $5.442,80 \mathrm{~kW}$ de exergia na forma de vapor para processo. $\mathrm{O}$ consumo de energia da unidade de processamento de palma pode variar de 850 à 1.900 $\mathrm{kWh}$, de acordo com a Tabela 1. Portanto, a planta de cogeração associada à unidade de extração de óleo de palma e palmiste gera um excedente mínimo de $1.487,69 \mathrm{kWh}$.

\subsection{Análise Exergoeconômica}

Os balanços de custos exergéticos para cada componente do sistema são mostrados nas Equações $(14,15$ e 16). Onde os termos $C i$ (R\$/hora) representam o produto da exergia $E i$ da corrente $i$ pelo seu respectivo custo exergético específico $c i$.

$$
\begin{aligned}
& C_{6}=C_{2}+C_{3}+C_{w, \text { caldeira }}+Z_{\text {caldeira }} \\
& C_{7}=C_{6}+Z_{\text {tubula理es }} \\
& C_{w, \text { turbina }}+C_{8}=C_{7}+Z_{\text {turbina }}
\end{aligned}
$$

O custo do combustível foi considerado como 5\% do preço médio do CFF de palma no Brasil no ano de 2015 (IBGE, 2017), que corresponde à R $\$ 0,012$ por kg. A corrente 3, apesar de apresentar exergia desprezível, apresenta custo. Portanto, o valor de $C_{3}$ foi estimado pelo produto de sua vazão (Tabela 3) com o custo da água para indústria no Pará, $8,02 \mathrm{R} \$ / \mathrm{m}^{3}$ (CONSANPA, 2017). A utilização do estado do Pará como referência é devido a este ser o principal estado produtor de palma no Brasil (IBGE, 2017). Já custo do fluxo 5 foi desprezado, pois o principal custo associado à liberação dos gases de combustão para atmosfera é referente ao consumo de energia pelo sistema de depuração da caldeira, que já está contabilizado. A Tabela 5 apresenta os parâmetros utilizados para o cálculo dos os custos operacionais e capitais dos equipamentos $(Z)$ através das Equações 10 à 13.

Na Tabela 6 é possível visualizar um maior detalhamento do sistema e dos investimentos. Foram estimados 30 metros de tubulações de vapor entre a caldeira e a turbina. 
Tabela 5: Determinação das taxas de custos dos componentes.

\begin{tabular}{l|ccc}
\hline \multicolumn{1}{c}{ Caldeira } & Tubulações & Turbina \\
\hline $\mathrm{I}(\mathrm{R} \$)$ & $\mathrm{R} \$ 12.300 .000,00$ & $\mathrm{R} \$ 18.543,26$ & $\mathrm{R} \$ 4.250 .000,00$ \\
$\mathrm{r}(\%)$ & 13 & 13 & 13 \\
$\mathrm{n}($ anos $)$ & 20 & 10 & 20 \\
$\emptyset(\%)$ & 6 & 6 & 6 \\
$\mathrm{t}_{\mathrm{op}}$ (horas) & 5.280 & 5.280 & 5.280 \\
$\mathrm{~V}_{\mathrm{R}}(\mathrm{R} \$)$ & 0 & 0 & 0 \\
$\mathrm{Z}(\mathrm{R} \$$ /hora $)$ & 351,52 & 0,69 & 121,46 \\
\hline
\end{tabular}

Tabela 6: Investimentos do projeto de cogeração.

\begin{tabular}{llrr}
\hline Turbina de Contrapressão & Investimento inicial (R\$) \\
\hline $\begin{array}{l}\text { Conjunto Turbo-Redutor (completo com tanque de óleo } \\
\text { integrado). Sistema de proteção, controle e acessórios. }\end{array}$ & R $\begin{array}{l}2.000 .000,00 \\
\text { Gerador 4,5MVA e painéis }\end{array}$ & $\mathrm{R} \$ 1.800 .000,00$
\end{tabular}

Serviços de supervisão de instalação, comissionamento, partida

$\mathrm{R} \$ 450.000,00$ e operação assistida da turbina.

Subtotal

$\mathrm{R} \$ 4.250 .000,00$

Caldeira Aquatubular Investimento inicial $(\mathrm{R} \$)$

Caldeira aquatubular vertical - $30.000 \mathrm{~kg}$ vapor/hora - pressão $\mathrm{R} \$ 12.300 .000,00$ de trabalho $42 \mathrm{kgf} / \mathrm{cm}^{2}$ - temperatura do vapor $420^{\circ} \mathrm{C}$. Sistemas de bombas e tratamento de água. Sistema de controle e automação. Startup, instalação e Treinamento.

Subtotal $\mathrm{R} \$ 12.300 .000,00$ Tubulações adicionais Investimento inicial (R\$)

Tubulações de aço carbono e válvulas $\mathrm{R} \$ 4.699,30$

Isolamento térmico - lã de rocha

$\mathrm{R} \$ 2.869,38$

Instalação

$\mathrm{R} \$ 10.974,58$

Subtotal

$\mathrm{R} \$ 18.543,26$

Total $\mathrm{R} \$ 16.568 .543,26$

O custo da instalação das tubulações foi estimados em $45 \%$ dos custos dos equipamentos adquiridos (tubulações e isolamento térmico), seguindo as recomendações de Bejan et al. (1996). Os demais custos foram estimados junto a fornecedores dos 
equipamentos. Não estão incluídos custos com frete e construções civis para acomodar a unidade de cogeração.

Para realizar a partição dos custos foi considerado o critério da extração (ABUSOGLU; KANOGLU, 2009), ou seja, que o custo da corrente de vapor produzida à alta pressão é o mesmo do vapor de baixa pressão, e que os demais custos devem ser atribuídos à potência gerada pela turbina. Dessa forma, os custos específicos encontrados para o vapor e eletricidade foram, respectivamente, de 12,18 R\$/ton e 124,12 R\$/MWh.

O principal benefício da cogeração para a indústria é a redução de custos com compra de energia elétrica. Levando em conta o estado do Pará, o custo da eletricidade rural é de 395,88 R\$/MWh (CELPA, 2016). Sobre essa alíquota há incidência de 25\% de Imposto de Circulação de Mercadorias e Serviços (ICMS); 1,65\% de Programas de Integração Social e de Formação do Patrimônio do Servidor Público (PIS) e 7,60\% Contribuição para Financiamento da Seguridade Social (COFINS). Sendo o custo final da energia de 602,10 $\mathrm{R} \$ \mathrm{MWh}$, seguindo a metodologia de cálculo da Agência Nacional de Energia Elétrica (2008). Portanto, há uma redução de R\$ 477,98 a cada MWh consumido pelas unidade industrial.

Considerando que a unidade de processamento de palma, assim como a planta de cogeração, opera 5280 horas por ano, isso pode significar uma economia de até $\mathrm{R} \$$ 4.795.083,92. Além disso, o excedente de $1.487,69 \mathrm{kWh}$ poderia gerar créditos para serem utilizados no abatimento de outros setores da empresa, como administrativo-financeiro e agrícola, via minigeração distribuída ${ }^{2}(75$ a $5000 \mathrm{~kW})$.

\section{CONCLUSÕES}

A análise exergoeconômica, utilizando a metodologia SPECO, foi aplicada à um projeto de cogeração com a biomassa residual da palma a fim de se avaliar o custo de produção de vapor e eletricidade. Os custos encontrados para o vapor e eletricidade foram, respectivamente, de 12,18 R \$/ton e 124,12 R \$/MWh. Ao se comparar o custo de produção da energia com o custo de sua compra, constatou-se que a implantação da cogeração pode gerar uma economia anual de $\mathrm{R} \$$ 4.795.083,92 para a unidade de processamento de palma deste estudo

Esses resultados evidenciam que mesmo projetos de cogeração de pequeno porte podem trazer economia significativa para indústrias que dispõem de biomassas residuais com bom poder calorífico.

\section{AGRADECIMENTOS}

Os autores agradecem ao Conselho Nacional de Pesquisa e Desenvolvimento Tecnológico, CNPq, pelo suporte financeiro (bolsa de iniciação científica para o primeiro autor - processo de concessão número 126390/2016-9).

${ }^{2}$ RESOLUÇÃO NORMATIVA No 482, DE 17 DE ABRIL DE 2012. 


\section{REFERÊNCIAS}

ABUSOGLU, A.; KANOGLU, M. Exergoeconomic analysis and optimization of combined heat and power production: A review. Renewable and Sustainable Energy Reviews, v. 13, n. 9, p. 2295-2308, 2009.

Agência Nacional de Energia Elétrica - ANEEL. POR DENTRO DA CONTA DE LUZ: Utilidade Pública. Brasília, 2008. Disponível em:< http://www2.aneel.gov.br/arquivos/pdf/catilha_1p_atual.pdf $>$. Acesso em Março de 2017.

ARRIETA, F. R. P. et al. Cogeneration potential in the Columbian palm oil industry: Three case studies. Biomass and Bioenergy, v. 31, n. 7, p. 503-511, 2007.

BEJAN, A.; TSATSARONIS, G.; MORAN, M.; Thermal design \& optimization; John Wiley \& Sons, Inc., NY, 542 p., 1996.

CESPEDES, J; JUNIOR, S; Análise Termoeconômica de Plantas de Cogeração. Revista Brasileira de Engenharia Química, São Paulo: vol.17, n 4, p. 21-27, Dez. 97/Jan. 98.

CELPA. Centrais Elétricas do Pará. Cobraça de Tarifas. Disponível em: < http://www.celpa.com.br/residencial/informacoes/cobranca-de-tarifas $>$. Acesso em março de 2017.

CONSANPA. Companhia de Saneamento do Pará. TARIFAS - CONSANPA. Disponível em: $<$ http://www.cosanpa.pa.gov.br/index.php/acesso-a-informacao/2013-06-20-08-5212>. Acesso em março de 2017.

HUSAIN, Z.; ZAINAL, Z. A.; ABDULLAH, M. Z. Analysis of biomass-residue-based cogeneration system in palm oil mills. Biomass and Bioenergy, v. 24, n. 2, p. 117-124, 2002.

IBGE. Instituto Brasileiro de Geografia e Estatística. Sistema IBGE de recuperação automática. Produção Agrícola Municipal. Disponível em: $<$ http://www.sidra.ibge.gov.br/bda/Tabela/listabl.asp?c=1613\&z=t\&o=11>. Acesso em março de 2017.

JÚNIOR, J. F. Dendê : Manejo e Uso dos Subprodutos e dos Resíduos. Embrapa Amazônia Oriental Belém, p. 40, 2006.

KEÇEBAŞ, A. The Modified Productive Structure Analysis of Afyon Geothermal District Heating System for Economic Optimization. INTERNATIONAL JOURNAL of RENEWABLE ENERGY RESEARCH, v. 3, n. 1, p. 60-67, 2013.

KIM, S.-M. et al. Exergoeconomic analysis of thermal systems. Energy, v. 23, n. 5, p. 393406, 1998.

KLEIN, S.A., ALVARADO, F.L.; EES - Engineering Equation Solver; F-Chart Software, Middleton, WI; 1995.

KWAK, H. Y.; KIM, D. J.; JEON, J. S. Exergetic and thermoeconomic analyses of power plants. Energy, v. 28, n. 4, p. 343-360, 2003.

LAZZARETTO, A.; TSATSARONIS, G. On the calculation of efficiencies and costs in thermal systems. In: Aceves SM, Garimella S, Peterson R, editors. Proceedings of the ASME advanced energy systems division, vol. 39. Anais...1999

LAZZARETTO, A.; TSATSARONIS, G. SPECO: A systematic and general methodology for calculating efficiencies and costs in thermal systems. Energy, v. 31, n. 8-9, p. 1257-1289, 2006.

LOZANO, M. A.; VALERO, A. Theory of the exergetic cost. Energy, v. 18, n. 9, p. 939- 
954, 1993.

MORAN, M. J. Availability analysis: a guide to efficient energy use. Englewood Cliffs, NJ: Prentice-Hall, 1982.

MORAN, M. J. SHAPIRO, H. N. Princípios de Termodinâmica para Engenharia. $6^{\mathrm{a}}$ ed. Rio de Janeiro: LTC, 2009.

PRASERTSAN, S.; PRASERTSAN, P. Biomass residues from palm oil mills in Thailand: An overview on quantity and potential usage. Biomass and Bioenergy, 1996.

ROSEN, M. A.; DINCER, I. Thermoeconomic analysis of power plants: An application to a coal fired electrical generating station. Energy Conversion and Management, v. 44, n. 17, p. 2743-2761, 2003.

SZARGUT J., MORRIS, D.R., STEWARD, F.R.; Exergy Analysis of Thermal, Chemical and Metallurgical Process; Hemisphere Publishing Corporation; 332 p.; New York; 1988

TALIB, N.; AMIN, M.; MAJID, A. THERMODYNAMIC ANALYSIS ON OIL PALM BIOMASS. ARPN Journal of Engineering and Applied Sciences, v. 11, n. 22, p. 12959-12966, 2016.

TSATSARONIS, G. Definitions and nomenclature in exergy analysis and exergoeconomics. Energy, v. 32, n. 4, p. 249-253, 2007.

UEMURA, Y. et al. Torrefaction of oil palm wastes. Fuel, v. 90, n. 8, p. 2585-2591, 2011.

USDA. United States Department of Agriculture. Oilseeds: World Market and Trade. 2016. Disponível em $<$ http://apps.fas.usda.gov/psdonline/circulars/oilseeds.pdf $>$. Acesso em: 05 nov. 2016.

YUSOFF, S. Renewable energy from palm oil - Innovation on effective utilization of waste. Journal of Cleaner Production, v. 14, n. 1, p. 87-93, 2006. 